Article

\title{
Fabrication of a Conjugated Fluoropolymer Film Using One-Step iCVD Process and its Mechanical Durability
}

\author{
Hyo Seong Lee ${ }^{1}$, Hayeong Kim $^{2}$, Jeong Heon Lee ${ }^{1}$ and Jae B. Kwak ${ }^{1, *}$ \\ 1 Department of Mechanical System and Automotive Engineering, Chosun University, 309 Pilmun-daero, \\ Dong-gu, Gwangju 61452, Korea \\ 2 Global Technology Center, Samsung Electronics Co., Ltd., 129 Samsung-ro, Yeongtong-gu, \\ Suwon-si 16677, Korea \\ * Correspondence: jaekwak@chosun.ac.kr; Tel.: +82-62-230-7095
}

Received: 12 June 2019; Accepted: 4 July 2019; Published: 8 July 2019

\begin{abstract}
Most superhydrophobic surface fabrication techniques involve precise manufacturing process. We suggest initiated chemical vapor deposition (iCVD) as a novel CVD method to fabricate sufficiently durable superhydrophobic coating layers. The proposed method proceeds with the coating process at mild temperature $\left(40^{\circ} \mathrm{C}\right)$ with no need of pretreatment of the substrate surface; the pressure and temperature are optimized as process parameters. To obtain a durable superhydrophobic film, two polymeric layers are conjugated in a sequential deposition process. Specifically, 1,3,5,7-tetravinyl-1,3,5,7-tetramethylcyclotetrasiloxane (V4D4) monomer is introduced to form an organosilicon layer (pV4D4) followed by fluoropolymer formation by introducing $1 \mathrm{H}, 1 \mathrm{H}$, 2H, 2H-Perfluorodecyl methacrylate (PFDMA). There is a high probability of covalent bond formation at the interface between the two layers. Accordingly, the mechanical durability of the conjugated fluoropolymer film (pV4D4-PFDMA) is reinforced because of cross-linking. The superhydrophobic coating on soft substrates, such as tissue paper and cotton fabric, was successfully demonstrated, and its durability was assessed against the mechanical stress such as tensile loading and abrasion. The results from both tests confirm the improvement of mechanical durability of the obtained film.
\end{abstract}

Keywords: initiated chemical vapor deposition (iCVD); superhydrophobic; fluoropolymer

\section{Introduction}

Superhydrophobic surfaces attracts considerable research attention as they are used in many industrial applications, including water repellents, antifouling, and self-cleaning surfaces [1-12]. There are many methods and techniques to produce superhydrophobic surfaces such as metal etching $[2,3]$, sol-gel processing [4], dip-spraying [5], electro-spray and spinning [6,11], combining solution and phase inversion process [7,8], solution method [9], ultrasound-assisted method [10], and plasma-based method [12]. However, most of these techniques are only suitable for nanostructures with fine surface roughness and are further limited to specific substrates such as metals. A hierarchical structure is always better for a superhydrophobic surface because it can reduce the contact area between the water and the surface. This surface can be obtained using a combination of the topographical properties of the surface texture and the chemical properties of low surface energy. To demonstrate this, Zhou et al. have synthesized nanostructured $\mathrm{ZnO}$ film with super-hydrophobicity using a chemical vapor deposition (CVD) method [13].

In comparison with other methods, the CVD process can produce large area of homogeneous thin films. However, it is not readily available for flexible substrates such as cotton fabrics and papers because it requires the use of high temperature and high vacuum pressure. For commercial purposes, 
a superhydrophobic surface needs to be mechanically and chemically durable. For instance, Zhou et al. fabricated a durable superhydrophobic polyester fabric with fluoroalkyl silane-modified silicone rubber, which is a nanoparticle composite, using the dip coating method [14]. Yan et al. also fabricated hydrophobic and hydrophilic surface of cotton fabric using a plasma-induced graft method [15]. Recently, Heydari Gharahcheshmeh and Gleason reported the fabrication of an antifouling surface on conductive polymer film via the oxidative chemical vapor deposition (oCVD) process that improves signal-to-noise ratio of the neural recording electrodes against blood or tissue contamination [16]. In this study, we use an iCVD process to generate fluoropolymer film for super hydrophobicity because this is relatively simple process in comparison with the previous methods. Unlike the conventional CVD process, the iCVD is achievable at a low temperature and vacuum pressure and shows excellent step coverage at high aspect ratio structure [17].

Figure 1 shows the iCVD process. The process begins with canisters containing an initiator $\left(\mathrm{I}_{2}\right)$ and one or more monomers $(\mathrm{M})$, which are the building blocks of the desired polymer coating. These materials are vaporized, either by heating or reducing the air pressure, and are introduced simultaneously into a vacuum chamber with the substrate to be coated. Once vaporized, the initiator molecules are thermally decomposed upon the contact with a hot filament to become radicals $\left(\mathrm{I}^{*}\right)$. The radicals activate the vaporized monomer to link in chains that form polymers on the surface of the substrate kept at mild temperature $\left(25-40^{\circ} \mathrm{C}\right)$. This is a one-step, solvent-free process to grow polymer films uniformly onto complex substrates structures, regardless of the substrate material. The functional performance of the polymer thin film is attributed to the properties of the monomers used [18].

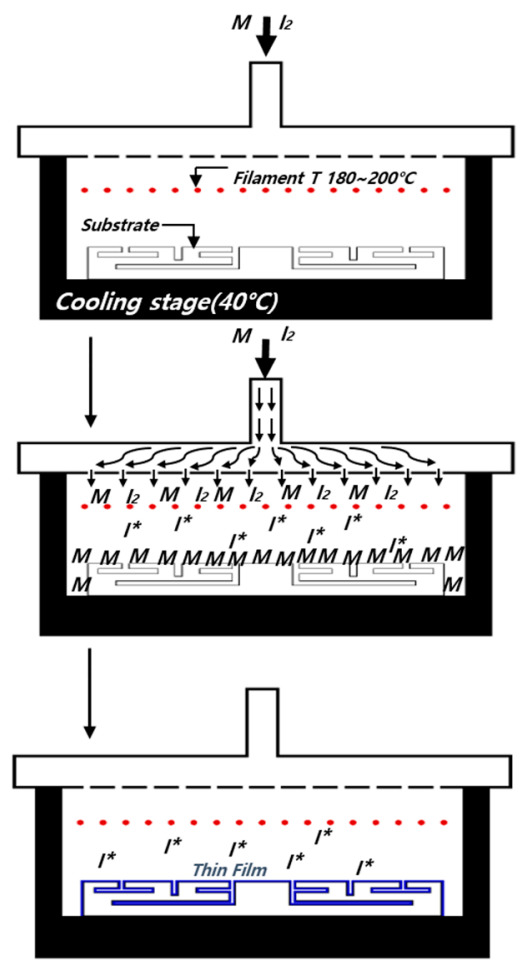

Figure 1. A schematic of iCVD process.

To obtain the fluoropolymer film with the iCVD process, tert-butyl peroxide (TBPO) and $1 \mathrm{H}$, $1 \mathrm{H}, 2 \mathrm{H}, 2 \mathrm{H}$-perfluorodecyl acrylate (PFDA) are typically used as the initiator and the monomer, respectively [19-22]. In particular, $1 \mathrm{H}, 1 \mathrm{H}, 2 \mathrm{H}, 2 \mathrm{H}$-perfluorodecyl methacrylate (PFDMA) is of great interest because of its exceptionally low surface energy $\left(3.5 \mathrm{mN} \cdot \mathrm{m}^{-1}\right)$, similar to PFDA and more efficient commercialization possibilities $[23,24]$. These monomers are synthesized in a one-step iCVD process and show more than $150^{\circ}$ of water contact angle. Previously, mechanical and chemical robustness 
of the iCVD superhydrophobic coating had not been studied. Therefore, 1,3,5,7-tetravinyl-1,3,5,7tetramethylcyclotetrasiloxane (V4D4) was introduced as a cross linker, which improves mechanical strength. Finally, a conjugated polymer film was obtained with a successive deposition of an organosilicon polymer, pV4D4 (poly-tetramethylcyclotetrasiloxane) and a fluoropolymer, pPFDMA (poly-perfluorodecyl methacrylate) using the customized iCVD reactor. Because pPFDMA is strongly bound on the pV4D4 layer, the durability of the conjugated film (pV4D4-PFDMA) improves due to increasing adhesion strength between the substrate and the fluoropolymer film. In this study, only pPFDMA, pV4D4-PFDA, and pV4D4-PFDMA were synthesized with iCVD, after which the durability of the each film was evaluated using the rubbing test. In addition, infiltration capability was examined after coating the folded copper sheet.

\section{Experiment}

\section{1. iCVD Process Basics}

The iCVD reaction mechanism is initiated by pyrolysis of the initiator to radicalize non-covalent electrons to bond with the vinyl group of the monomer and to grow the polymer thin film through the sequential chain bonding $[25,26]$. Notably, the initiator TBPO is radicalized through the pyrolysis and polymerized with the vinyl groups of monomers such as V4D4 and PFDMA, resulting in thin film growth. As shown in Equations (1)-(3), the parameter of iCVD process is defined as an optimal value of $P_{\mathrm{M}} / P_{\text {Sat }}$. The $P_{\mathrm{M}}$ and $P_{\text {Sat }}$ values are derived from the Antoine Equations (1) and (2), where $P_{\mathrm{M}}$ is the ratio of the incoming gas amount controlled by the vaporization temperature of the monomer and the initiator, and the $P_{\text {Sat }}$ value is determined by monomer's characterization and the temperature of the substrate $\left(T_{\text {substrate }}\right)$. The optimized $P_{\mathrm{M}} / P_{\text {Sat }}$ is between 0 and 1 , which is the rate of polymerization. When $P_{\mathrm{M}} / P_{\text {Sat }}$ is close to 0 , the growth rate is very slow. When it is close to 1 , condensation occurs instead of polymerization. Also, the duration of the deposition affects the thickness of the polymerized film. Optimized $P_{\mathrm{M}} / P_{\text {Sat }}$ varies depending on monomers.

$$
\begin{gathered}
P_{\mathrm{M}}=P_{\text {Chamber }} \times \frac{F_{\mathrm{M}}}{F_{\mathrm{M}}+F_{\mathrm{I}}} \\
\log P_{\text {Sat }}=A-\frac{B}{T_{\text {substrate }}} \\
0<\frac{P_{\mathrm{M}}}{P_{\text {Sat }}}<1
\end{gathered}
$$

where, $F_{\mathrm{M}}(\mathrm{sccm})$ is a gaseous monomer input flow, $F_{\mathrm{I}}(\mathrm{sccm})$ is a gaseous initiator input flow, $P_{\mathrm{M}}$ (mTorr) is the partial pressure of the monomer in the chamber, $P_{\text {Sat }}$ (mTorr) is the saturated vapor pressure at the $T_{\text {Substrate, }} P_{\text {Chamber }}$ (mTorr) is the total pressure of the chamber, and $T_{\text {Substrate }}\left({ }^{\circ} \mathrm{C}\right)$ is the temperature of substrate.

The customized iCVD reactor system uses canisters for monomers and the initiator. The canisters are heated to efficiently vaporize the materials into the vacuum chamber. Also, there are filaments arrayed in the vacuum chamber to instantly apply the elevated temperature for decomposing the initiator into radicals.

\subsection{Fabrication of Superhydrophobic Film and its Characteristics}

Table 1 shows the monomers and the initiator examined in this study. The deposition of pV4D4-PFDMA was performed in the iCVD reactor. V4D4 and PFDMA monomers were heated to 55 and $75{ }^{\circ} \mathrm{C}$, respectively, and TBPO was heated to $35^{\circ} \mathrm{C}$ through each canister. Each flow rate of the vapor was controlled by needle valves, and the flow was monitored by a pressure gauge installed at the inlet of the reactor. In this study, for all monomers and initiator, the vapor saturation ratio $\left(F_{\mathrm{M}}: F_{\mathrm{I}}\right)$ was maintained to be $1: 1$ as empirically optimized $P_{\mathrm{M}} / P_{\text {sat. }}$. First, V4D4 and TBPO were delivered into the reactor at $0.6 \mathrm{sccm}$ while maintaining $270 \mathrm{mTorr}$ vacuum pressure in the reactor. The substrate and the filaments 
temperature were held at 40 and $180^{\circ} \mathrm{C}$, respectively. Then, a homopolymer of $\mathrm{pV} 4 \mathrm{D} 4$ was grown from the surface of substrate. Once the desired thickness of $\mathrm{pV} 4 \mathrm{D} 4$ was obtained, the next vaporized PFDMA was introduced into the reactor together with V4D4 for 2-3 min. This period ensures strong adhesion at the film interface between the top pV4D4 layer and the bottom pPFDMA layer. Then, the flow of V4D4 was stopped, and the deposition of pPFDMA continued to the top layer. With PFDMA, only the vacuum pressure in the reactor was changed to 100 mTorr to maintain the optimal $P_{\mathrm{M}}$ value; other temperature settings remained the same. As a result, a conjugated fluoropolymer film was obtained as a superhydrophobic surface, as shown in Figure 2a. The film was sequentially stacked pV4D4 and pV4D4-PFDMA on a silicon wafer substrate. Figure $2 b$ shows the morphology of the fluoropolymer film; one can see about $5 \mu \mathrm{m}$ size fluorocarbon structures tangled from the top view. To confirm the super-hydrophobicity of film, the contact angle was measured with $50 \mu \mathrm{L}$ of deionized water droplets using a contact angle meter (SmartDrop, FEMTOBIOMED, Seongnam, Korea). As a result, the water contact angle of $150.1^{\circ} \pm 3.6^{\circ}$ was obtained while the sliding angle was only $8.5^{\circ}$. This means that the pV4D4-PFDMA film exhibits a highly non-stick super-hydrophobic surface.

Table 1. Chemical structures and functions of the initiator and the monomers.

Name (Abbreviation)
Chemical Formula
tert-butyl peroxide (TBPO)

Because the temperature of the substrate was kept at $40{ }^{\circ} \mathrm{C}$, relatively flexible substrates were considered such as paper and fabric. Accordingly, we choose tissue paper and cotton fabric as substrates and proceeded with the $\mathrm{PV} 4 \mathrm{D} 4-\mathrm{PFDMA}$ coating process in the iCVD reactor. At this time, the total thickness of the film was $200 \mathrm{~nm}$ so there were no significant changes in dimensions of the fiber structures. Both tissue and fabric are known to easily get wet. After coating, however, blue ink was dropped on the surface, and excellent repellency to the droplet was observed, as shown in Figure 3a,b. In addition, we performed SEM analysis (FE-SEM, S-4800, Hitachi, Tokyo, Japan), on both substrates before and after coating as shown in Figure $3 \mathrm{~b}-\mathrm{f}$, respectively. No noticeable changes were observed.

Another advantage of the iCVD process is that it shows ultra-step coverage on high aspect ratio of up to 1:40 for the opened area [27]. Therefore, we made a quantitative analysis of the capability of coating with shadow area. A manifolded copper sheet was subjected to the iCVD process and coated with $200 \mathrm{~nm}$ of the pV4D4-PFDMA, as seen in Figure 4a. The thickness and the width of the copper sheet were 0.02 and $25 \mathrm{~mm}$, respectively, and it was firmly folded seven times, as seen in Figure $4 \mathrm{~b}$. Next, the gap between each surface was measured about $0.1 \mathrm{~mm}$. The pure copper sheet had a hydrophilic surface, as seen in Figure 4c; therefore, water droplets on various places of the unfolded sheet indicated the successful coating, as seen in Figure 4d. Additionally, Fourier transform infrared spectra (Nicolet 6700 FT-IR Spectrometer, Thermo Scientific, Waltham, MA, USA) analysis has proven an infiltration capability, as shown in Figure 4e. Although the peaks are not so obvious due to an extremely high aspect ratio for the shadow area, the result verifies the presence of the fluorocarbon functional group. 


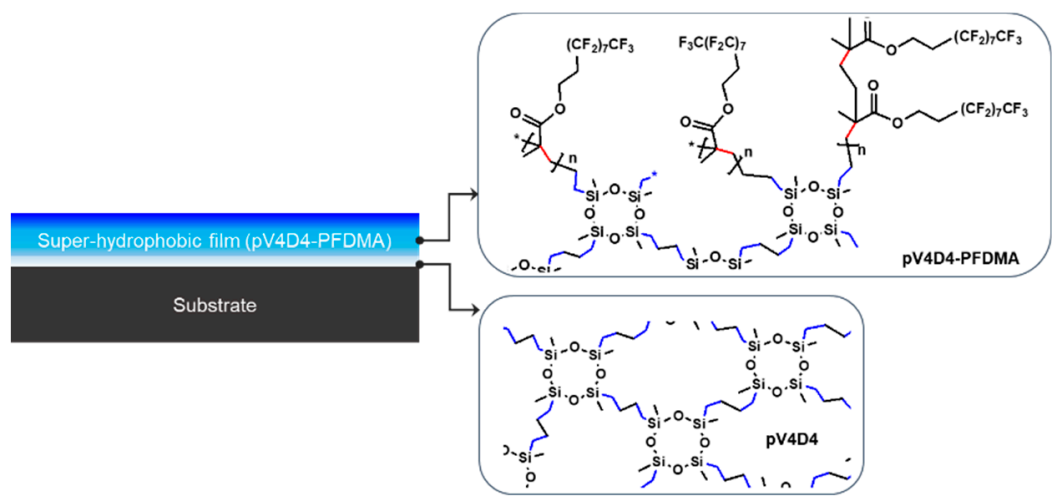

(a)

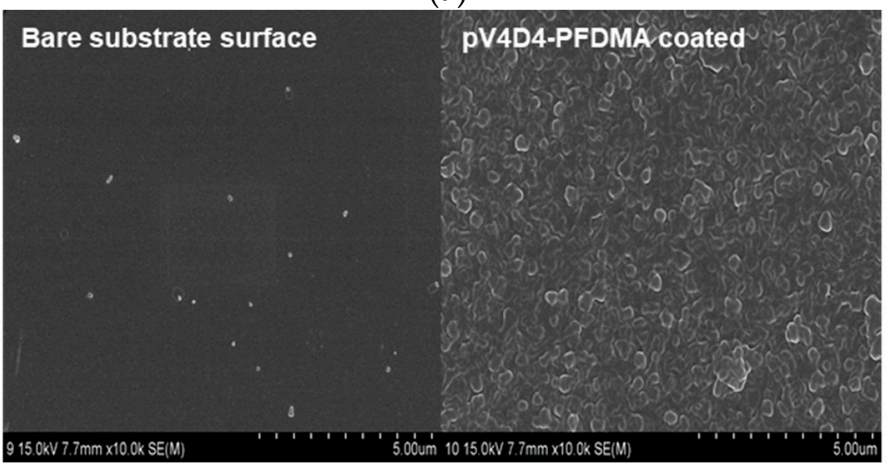

(b)

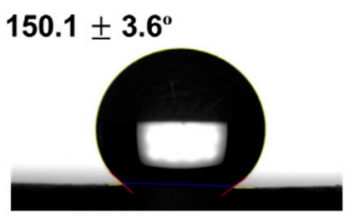

Water contact angle

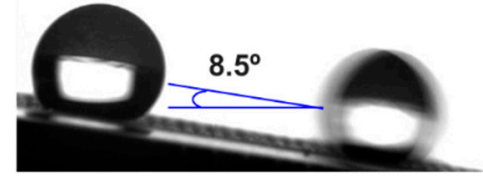

Sliding angle

(c)

Figure 2. (a) A schematic of the fluoropolymer (pV4D4-PFDMA) coating; (b) surface morphology using scanning electron microscope (FE-SEM, S-4800, Hitachi, Tokyo, Japan) imaging before and after coating on wafer substrate; (c) static and dynamic water contact angles of the pV4D4-PFDMA surface.
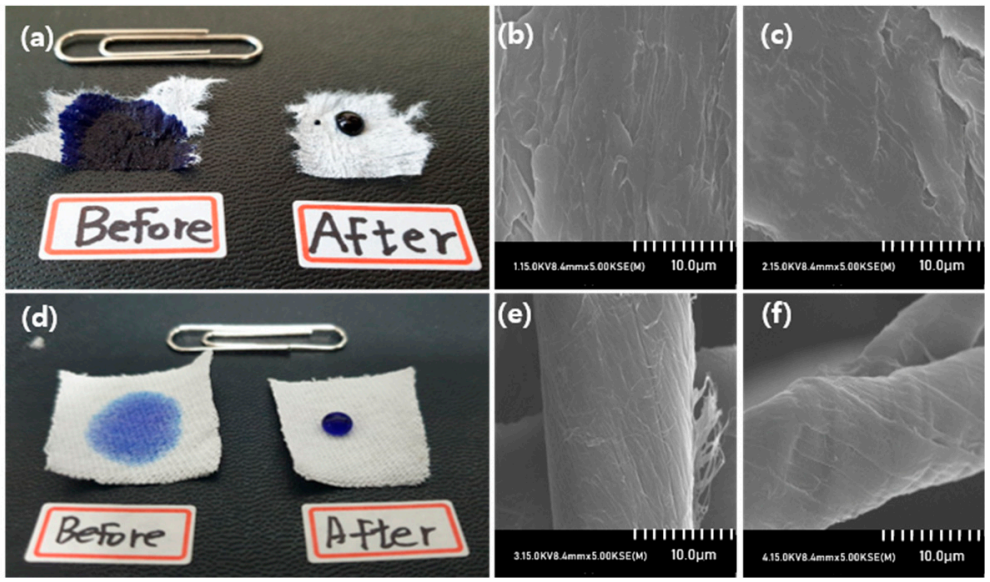

Figure 3. (a) Blue ink droplet on tissue paper before and after pV4D4-PFDMA coating; (b) SEM image of the tissue paper; (c) SEM image of the tissue structure after coating; (d) blue ink droplet on cotton fabric before and after pV4D4-PFDMA coating; (e) SEM image of the cotton fabric; and (f) SEM image of the cotton fabric after coating. 

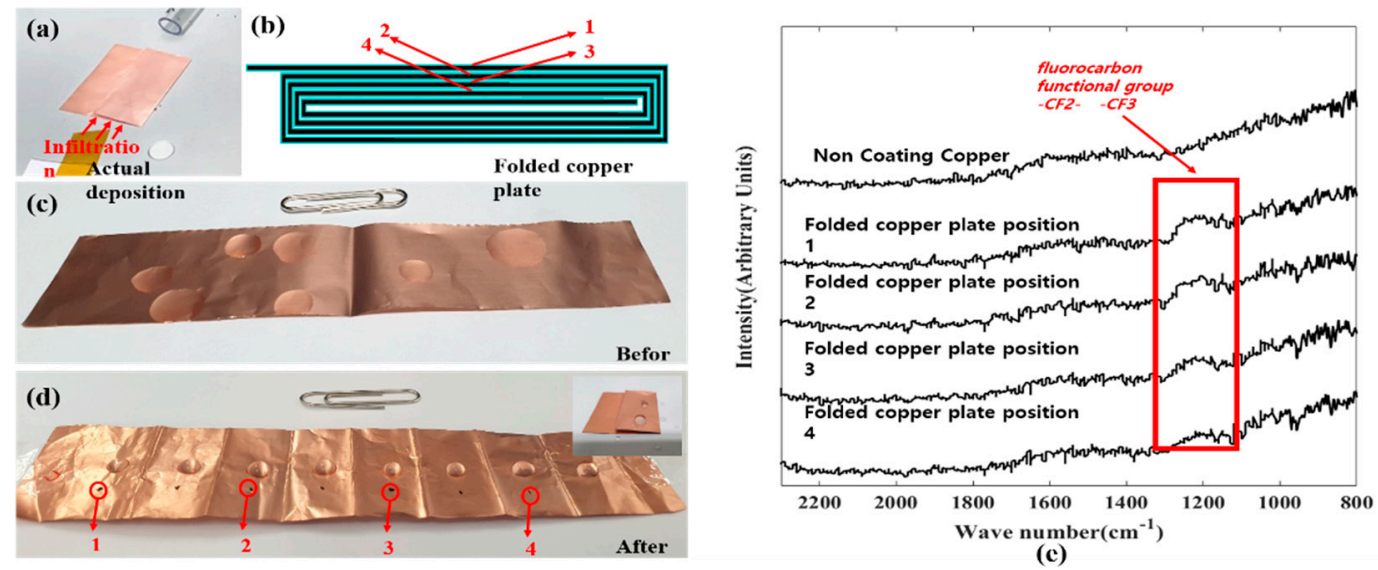

Figure 4. (a) A manifolded copper sheet in the iCVD reactor; (b) schematic of the folded copper sheet and FT-IR Spectrometer measurement locations; (c) water droplets on bare copper sheet; (d) water droplets on unfolded copper sheet after coating; and (e) FT-IR analysis before and after coating.

\subsection{Examination of Durability}

The development of a thin film that is resistant to various mechanical stresses is the key for successful commercialization of the superhydrophobic surfaces. The proposed film was obtained using the iCVD process, with a mechanically robust organosilicon polymer and a fluoropolymer layer. The two layers were covalently adhered to form a conjugated polymer structure. We evaluated the durability of the proposed coating against tensile and abrasion tests.

To compare the mechanical durability against deformation under external force between pPFDMA and pV4D4-PFDMA, we examined optical microscopic images of the cracks developed when the tensile load is applied. The pPFDMA $(500 \mathrm{~nm})$ and pV4D4 $(100 \mathrm{~nm})$-PFDMA $(500 \mathrm{~nm})$ were deposited on the elastomer film (50 $\mu \mathrm{m}$ thickness) and subjected to tensile tests using Instron tester (5960 Series, INSTRON, Chicago, IL, USA) according to ASTM standard [28], as shown in Figure 5a. Tensile tests for each thin film were proceeded with the strain rate of $2.5 \mathrm{~mm} / \mathrm{min}$, and the obtained stress-strain plots were compared with the bare elastomer film, pPFDMA coated, and pV4D4-PFDMA coated, as seen in Figure 5d. According to the result, the pPFDMA coating shows the tensile strength was similar to that of the bare elastomer film; surface cracking was observed when the strain reached about $60 \%$, as seen in Figure 5b,c. However, for pV4D4-PFDMA coating, crack occurrence was observed at 110\% of strain, indicating an improvement of the adhesion strength between the elastomer film and the fluoropolymer film, resulting in eventual enhancement of the global tensile strength.
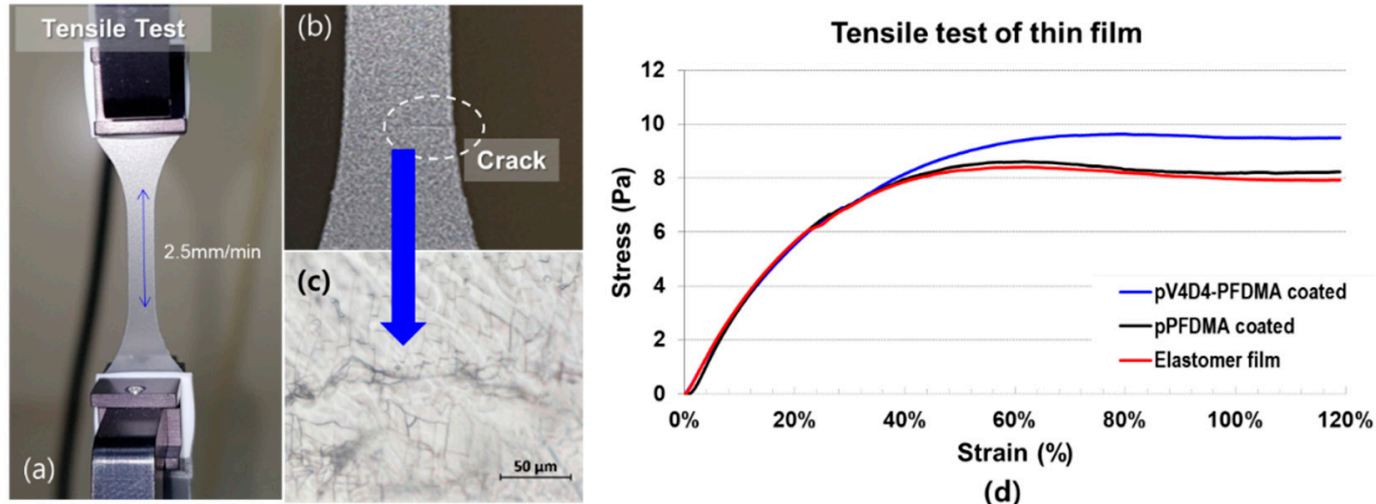

(d)

Figure 5. Tensile test of the elastomer film with superhydrophobic coating: (a) tensile test set-up; (b) surface cracks occurred during tensile loading; (c) microscopic image of the surface cracks; and (d) stress-strain curves. 
The combined pV4D4 and pPFDMA film showed better stability and the super-hydrophobicity because of the covalent bonding between the interlayers. In this structure, benzene ring of the V4D4 acts as a cross linker, protecting the conjugated pV4D4-PFDMA from external stresses that normally occur in daily life. Accordingly, we evaluated the resistance in the abrasion test (CT-RB Series, CORETECH, Uiwang, Korea) and compared pPFDMA (500 nm) only, pV4D4 (100 nm)-PFDA (500 nm), and pV4D4 (100 nm)-PFDMA (500 nm). For the abrasion test, these films were deposited onto the SUS304 plate; the comparison was made using a contact angle meter. Figure 6a shows the abrasion test set-up. The coated sample was placed and firmly fixed in the sample holder. Then, the cotton-covered ball-shape tip was released to contact the top surface of the coated sample. $1 \mathrm{kgf}$ weight was loaded on the tip, which was repeatedly rubbed on the surface left- and right-hand side throughout 3000 cycles. The contact angle was measured at every five cycles in the beginning and intermittently after hundreds of cycles. In abrasion test, the coated layer appeared to be scratched on the surface, as seen in Figure $6 \mathrm{c}$ and contact angle was gradually decreased. Figure $6 \mathrm{~d}$ shows the comparison of the contact angle change for each film. According to the results, pPFDMA only showed the lowest abrasion resistance indicating drastic reduction of the contact angle within less than 10 cycles. However, the other two fluoropolymer films with pV4D4 conjugation withstood 200 cycles of rubbing and retained contact angle of $120^{\circ}$, which is considered as sufficiently high hydrophobicity. The proposed pV4D4-PFDMA showed the highest abrasion resistance, retaining $120^{\circ}$ of the contact angle after 3000 cycles.

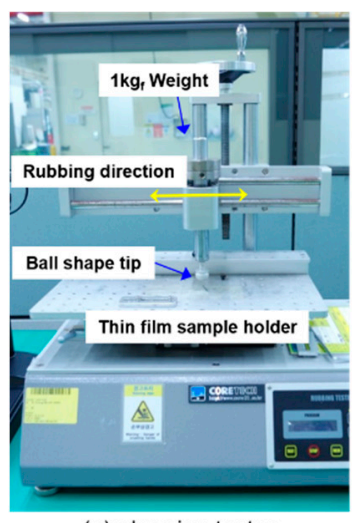

(a) abrasion tester

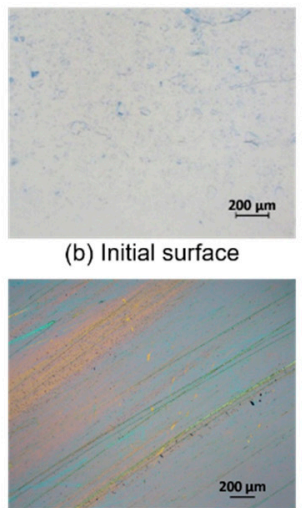

(c) tested surface

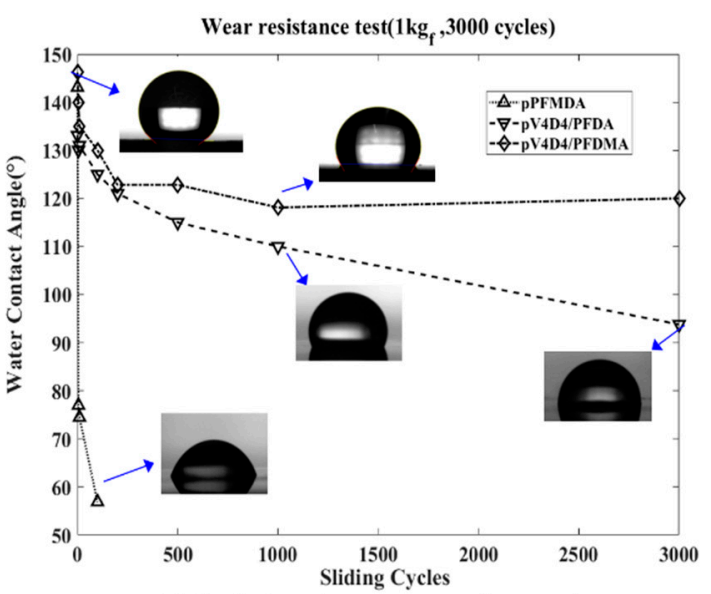

(d) Contact angle measurement comparison

Figure 6. (a) Abrasion test set-up; (b) microscopic image of the initial surface; (c) microscopic image of the tested surface; and (d) contact angle measurements.

\section{Conclusions}

A fluoropolymer fabricated using the iCVD process has great advantages such as cost efficiency and high functionality when compared with other surface modification solutions to obtain super hydrophobicity. In this study, we examined in detail parametric optimization of the iCVD process and successfully fabricated a new super-hydrophobic film conjugated with V4D4 and PFDMA. The proposed superhydrophobic film was applied to tissue paper and cotton fabric and demonstrated great liquid repellency. In addition, high infiltration capability of the iCVD process was discussed using manifolded copper sheet. These results provide enough insight for industrial applications in which superhydrophobic surfaces are needed.

A conjugated film (pV4D4-PFDMA) was achieved by adding the pV4D4 layer before the introduction of the pPFDMA and showed exceptional stability and durability. The pV4D4 significantly enhances mechanical stability of the pPFDMA as it allowed for both monomers to flow into the reactor. Therefore, the fluoropolymer was reinforced by binding the organosilicon layer. We evaluated the mechanical and chemical robustness of the proposed film. First, the tensile test was performed using the deposition on the elastomer film; it showed improvement both in terms of the tensile strength and 
delay in surface cracking. Second, the mechanical abrasion test was performed and the proposed film showed better rubbing resistance when compared with other films.

The deposition process is applicable to various types and complex shapes of the substrates without the need of surface pretreatment; is also allows for improved adhesion between the coated film and the substrate. We found that the proposed superhydrophobic film obtained in the iCVD process provides an industrial grade of low surface energy with sufficient durability against various mechanical stresses. In addition, optimization of iCVD process can be further studied to enhance mechanical properties.

Author Contributions: Conceptualization, J.B.K.; Data Curation, H.K.; Formal Analysis, J.H.L.; Investigation, H.S.L. and J.B.K; Methodology, H.K.; Writing_-Original Draft, H.S.L.; Writing-Review and Editing, J.B.K.

Funding: This research was funded by National Research Foundation of Korea (NRF) grant funded by the Korea government (MSIT) (No. NRF-2018R1C1B5045726) and Korea Institute for Advancement of Technology (KIAT) grant funded by the Korea Government (MOTIE) (P0002092, The Competency Development Program for Industry Specialist).

Conflicts of Interest: The authors declare no conflict of interest.

\section{References}

1. Wu, D.; Guo, Z. Robust and muti-repaired superhydrophobic surfaces via one-step method on copper and aluminum alloys. Mater. Lett. 2018, 213, 290-293. [CrossRef]

2. Latthe, S.S.; Sudhagar, P.; Devadoss, A.; Kumar, A.M.; Liu, S.; Terashima, C.; Nakata, K.; Fujishima, A. A mechanically bendable superhydrophobic steel surface with self-cleaning and corrosion-resistant properties. J. Mater. Chem. A 2015, 3, 14263-14271. [CrossRef]

3. He, J.; Wu, M.; Zhang, R.; Liu, J.; Deng, Y.; Guo, Z. A one-step hot-embossing process for fabricating a channel with superhydrophobic inner walls. J. Manuf. Process. 2018, 36, 351-359. [CrossRef]

4. Liu, S.; Liu, X.; Latthe, S.S.; Gao, L.; An, S.; Yoon, S.S.; Liu, B.; Xing, R. Self-cleaning transparent superhydrophobic coatings through simple sol-gel processing of fluoroalkylsilane. Appl. Surf. Sci. 2015, 351, 897-903. [CrossRef]

5. Latthe, S.S.; Sutar, R.S.; Kodag, V.S.; Bhosale, A.K.; Kumar, A.M.; Sadasivuni, K.K.; Xing, X.; Liu, S. Self-cleaning superhydrophobic coatings: Potential industrial applications. Prog. Org. Coat. 2019, 128, 52-58. [CrossRef]

6. Li, Y.; Men, X.; Zhu, X.; Ge, B.; Chu, F.; Zhang, Z. One-step spraying to fabricate nonfluorinated superhydrophobic coatings with high transparency. J. Mater. Sci. 2016, 51, 2411-2419. [CrossRef]

7. Fang, Z.; Li, H.; Xia, M.; Liu, Y.; Zhang, Y.; Li, Y.; Jiang, X.; He, P. A facile method to fabricate super-hydrophobic surface with water evaporation-induced phase inversion of stearic acid. Mater. Lett. 2018, 223, 124-127. [CrossRef]

8. Zhang, J.; Xu, Z.; Mai, W.; Min, C.; Zhou, B.; Shan, M.; Li, Y.; Yang, C.; Wang, Z.; Qian, X. Improved hydrophilicity, permeability, antifouling and mechanical performance of PVDF composite ultrafiltration membranes tailored by oxidized low-dimensional carbon nanomaterials. J. Mater. Chem. A 2013, 1,3101-3111. [CrossRef]

9. Zhang, L.; Zuo, W.; Li, T. Controlled synthesis of bifunctional $\mathrm{PbWO}_{4}$ dendrites via a facile solution method at room temperature: Photoluminescence and superhydrophobic property. Mater. Sci. Semicond. Process. 2015, 39, 188-191. [CrossRef]

10. Perkas, N.; Amirian, G.; Girshevitz, O.; Gedanken, A. Hydrophobic coating of GaAs surfaces with nanostructured ZnO. Mater. Lett. 2016, 175, 101-105. [CrossRef]

11. Rivero, P.J.; Iribarren, A.; Larumbe, S.; Palacio, J.F.; Rodríguez, R. A comparative study of multifunctional coatings based on electrospun fibers with incorporated $\mathrm{ZnO}$ nanoparticles. Coatings 2019, 9, 367. [CrossRef]

12. Ellinas, K.; Tserepi, A.; Gogolides, E. Superhydrophobic fabrics with mechanical durability prepared by a two-step plasma processing method. Coatings 2018, 8, 351. [CrossRef]

13. Zhou, M.; Feng, C.; Wu, C.; Ma, W.; Cai, L. Superhydrophobic multi-scale ZnO nanostructures fabricated by chemical vapor deposition method. J. Nanosci. Nanotechnol. 2009, 9, 4211-4214. [CrossRef] [PubMed] 
14. Zhou, H.; Wang, H.; Niu, H.; Gestos, A.; Wang, X.; Lin, T. Fluoroalkyl silane modified silicone rubber/ nanoparticle composite: A super durable, robust superhydrophobic fabric coating. Adv. Mater. 2012, 24, 2409-2412. [CrossRef]

15. Yan, X.; Li, J.; Yi, L. Fabrication of $\mathrm{pH}$-responsive hydrophilic/hydrophobic Janus cotton fabric via plasma-induced graft polymerization. Mater. Lett. 2017, 208, 46-49. [CrossRef]

16. Heydari Gharahcheshmeh, M.; Gleason, K.K. Device fabrication based on oxidative chemical vapor deposition (oCVD) synthesis of conducting polymers and related conjugated organic materials. Adv. Mater. Interfaces 2019, 6, 1801564. [CrossRef]

17. Baxamusa, S.H.; Gleason, K.K. Thin polymer films with high step coverage in microtrenches by initiated CVD. Chem. Vap. Depos. 2008, 14, 313-318. [CrossRef]

18. Martin, T.P.; Lau, K.K.; Chan, K.; Mao, Y.; Gupta, M.; O’Shaughnessy, W.S.; Gleason, K.K. Initiated chemical vapor deposition (iCVD) of polymeric nanocoatings. Surf. Coat. Technol. 2007, 201, 9400-9405. [CrossRef]

19. Mao, Y.; Gleason, K.K. Positive-tone nanopatterning of chemical vapor deposited polyacrylic thin films. Langmuir 2006, 22, 1795-1799. [CrossRef]

20. Gupta, M.; Gleason, K.K. Initiated chemical vapor deposition of poly $(1 \mathrm{H}, 1 \mathrm{H}, 2 \mathrm{H}, 2 \mathrm{H}$-perfluorodecyl acrylate) thin films. Langmuir 2006, 22, 10047-10052. [CrossRef]

21. Ma, M.; Gupta, M.; Li, Z.; Zhai, L.; Gleason, K.K.; Cohen, R.E.; Rubner, M.F.; Rutledge, G.C. Decorated electrospun fibers exhibiting superhydrophobicity. Adv. Mater. 2007, 19, 255-259. [CrossRef]

22. Ma, M.; Mao, Y.; Gupta, M.; Gleason, K.K.; Rutledge, G.C. Superhydrophobic fabrics produced by electrospinning and chemical vapor deposition. Macromolecules 2005, 38, 9742-9748. [CrossRef]

23. Kim, J.H.; Park, S.K.; Lee, J.W.; Yoo, B.; Lee, J.K.; Kim, Y.H. Hydrophobic perfluoropolymer thin-film encapsulation for enhanced stability of inverted polymer solar cells. J. Korean Phys. Soc. 2014, 65, 1448-1452. [CrossRef]

24. Tsibouklis, J.; Graham, P.; Eaton, P.J.; Smith, J.R.; Nevell, T.G.; Smart, J.D.; Ewen, R.J. Poly(perfluoroalkyl methacrylate) film structures: surface organization phenomena, surface energy determinations, and force of adhesion measurements. Macromolecules 2000, 33, 8460-8465. [CrossRef]

25. Gleason, K.K. CVD Polymer: Fabrication of Organic Surfaces and Devices; WILET-VCH: Weinheim, Germany, 2015.

26. Moni, P.; Al-Obeidi, A.; Gleason, K.K. Vapor deposition routes to conformal polymer thin films. Beilstein J. Nanotechnol. 2017, 8, 723-735. [CrossRef] [PubMed]

27. Chan, K.; Gleason, K.K. A mechanistic study of initiated chemical vapor deposition of polymers: analyses of deposition rate and molecular weight. Macromolecules 2006, 39, 3890-3894. [CrossRef]

28. ASTM D882-18: Standard Test Method for Tensile Properties of Thin Plastic Sheeting; ASTM International: West Conshohocken, PA, USA, 2018.

(C) 2019 by the authors. Licensee MDPI, Basel, Switzerland. This article is an open access article distributed under the terms and conditions of the Creative Commons Attribution (CC BY) license (http://creativecommons.org/licenses/by/4.0/). 